\title{
MIN-MAX THEORY, WILLMORE CONJECTURE AND THE ENERGY OF LINKS
}

\author{
FERNANDO C. MARQUES AND ANDRÉ NEVES
}

Dedicated to the 60th anniversary of the founding of IMPA.

\begin{abstract}
In this paper we give an overview of some aspects of the min-max theory of minimal surfaces, and discuss recent applications to conformally invariant problems in Geometry and Topology. The goal is to explain what the proofs of the Willmore conjecture for surfaces and the Freedman-He-Wang conjecture for links share in common. This is based on joint work of the authors [19] and on joint work of I. Agol and the authors [1].
\end{abstract}

\section{Contents}

1. Introduction

2. The $2 \pi^{2}$ Theorem

3. Family for the Willmore problem 11

4. Family for the Möbius problem 16

References

\section{INTRODUCTION}

Minimal surfaces are among the most natural objects in Differential Geometry, and have been studied for the past 250 years since the pioneering work of Lagrange (1762). They are defined as surfaces that locally minimize the area, a variational principle satisfied precisely by surfaces with zero mean curvature. In this paper we give an overview of some min-max aspects of the theory and discuss recent applications to conformally invariant problems in geometry and topology. The goal is to explain what the proofs of the Willmore conjecture for surfaces and the Freedman-He-Wang conjecture for links share in common.

The problem of finding a closed embedded minimal surface in a compact Riemannian three-manifold is considerably difficult. One of the reasons is that in general these surfaces are not (globally) area-minimizing. They are critical points of the area functional, but usually admit ambient deformations that can decrease their area.

The first author was partly supported by CNPq-Brazil and FAPERJ. The second author was partly supported by Marie Curie IRG Grant and ERC Start Grant. 
The idea of producing minimal surfaces by min-max methods has its roots in the work of Birkhoff [5], who studied the problem of existence of closed geodesics in Riemannian 2-spheres. The case of convex surfaces had been previously studied by Poincaré [20] (see also [8]). Birkhoff's ideas were later refined and extended, leading to the celebrated theorems by Lyusternik and Schnirelmann [17] (existence of three closed geodesics in any Riemannian two-sphere) and by Lyusternik and Fet [16] (existence of a closed geodesic in any compact Riemannian manifold).

The first steps to attack the problem of existence of higher-dimensional minimal submanifolds were taken by Almgren ([2], [3]). He invented the notion of varifolds for that purpose, and developed a general scheme to produce minimal varieties in Riemannian manifolds. He left open the question of regularity of these objects, a problem that was tackled later by his $\mathrm{Ph}$.D. student Jon Pitts [21] in the important case of codimension one. Their combined works form what we refer to as the Almgren-Pitts min-max theory, whose basic application up until very recently was the following theorem:

1.1. Theorem. (Pitts' 81, [21]) Let $\left(M^{n}, g\right)$ be an $n$-dimensional compact Riemannian manifold, with $3 \leq n \leq 6$. Then there exists a smooth, embedded, closed minimal hypersurface

$$
\Sigma^{n-1} \subset M
$$

1.2. Remark. The restriction to dimensions $n \leq 6$ in the previous theorem is not necessary if we allow $\Sigma$ to have a singular set of codimension 7 , as it was later shown in [26].

We now describe, in an informal way, the min-max procedure used to construct the minimal hypersurface $\Sigma$ when $n=3$. For a detailed account of the min-max theory in this setting one can read [7].

Consider the class $\Pi_{1}$ of all nontrivial sweepouts $\{\Sigma(t)\}_{t \in[0,1]}$ of $M^{3}$ by closed surfaces. (The word surface has to be understood in a more general sense). In general, if one can write $\Sigma(t)=\partial \Omega(t)$, with $\Omega(t) \subset M, \Omega(0)=\emptyset$, in a continuous fashion, we say the sweepout $\{\Sigma(t)\}$ is nontrivial if $\Omega(1)=$ $M$. A good example of a nontrivial sweepout is obtained by choosing a Morse function $f: M \rightarrow \mathbb{R}$ with $f(M)=[0,1]$, and defining

$$
\Sigma(t)=\{x \in M: f(x)=t\} .
$$

The idea then is to try to minimize the largest area among the surfaces of a sweepout over all sweepouts in $\Pi_{1}$. This leads to the notion of width:

$$
W\left(\Pi_{1}\right)=\inf _{\{\Sigma(t)\} \in \Pi_{1}} \sup _{t \in[0,1]} \operatorname{area}(\Sigma(t)) .
$$

The minimal surface $\Sigma$ of Theorem 1.1 is constructed so to satisfy

$$
\operatorname{area}(\Sigma)=W\left(\Pi_{1}\right) \text {. }
$$


In some specific examples it is possible to determine which minimal surface is obtained, but in general this is a difficult problem. For instance, it is expected that if we replace the one-dimensional sweepouts by $k$-parameter families of surfaces then the Morse index of the minmax minimal surface should be bounded above by $k$. (See [18] and [32] for the particular case of positive Ricci curvature and $k=1$.) Although the Almgren-Pitts theory successfully applies to any number of parameters, the general bound on the index is not known.

If the ambient manifold is the three-dimensional unit sphere $S^{3}$ endowed with its canonical metric $\bar{g}$, then the minimal surface produced by min-max over $\Pi_{1}$ is, modulo ambient isometries, the equator

$$
\bar{\Sigma}=S^{3} \cap\left\{x_{4}=0\right\} .
$$

Indeed, the standard sweepout $\Sigma_{0}(t)=\left\{x_{4}=2 t-1\right\}, t \in[0,1]$, satisfies

$$
\sup _{t \in[0,1]} \operatorname{area}\left(\Sigma_{0}(t)\right)=4 \pi,
$$

telling us that $W\left(\Pi_{1}\right) \leq 4 \pi$. Since the great spheres are the only minimal surfaces of $S^{3}$ with area less than or equal to $4 \pi$ and $W\left(\Pi_{1}\right)>0$ by [2], the above claim follows. Notice that this is consistent with the fact that the Morse index of the equator is 1 .

In order to be more precise, we need the language of geometric measure theory where smooth submanifolds are replaced by integral currents (see [27]). For an intuitive grasp of the ideas here described, though, it is enough to think of currents as oriented submanifolds of class $C^{1}$ with integer multiplicities.

Given a compact Riemannian three-manifold $\left(M^{3}, g\right)$, we denote the space of integral two-currents with boundary zero in $M$ by $\mathcal{Z}_{2}\left(M^{3}\right)$, and the space of integral three-currents in $M$ by $I_{3}(M)$.

A sweepout of $M$ is a map $\Phi: I \rightarrow \mathcal{Z}_{2}(M)$, with $\Phi(0)=\Phi(1)=0$, continuous in the flat topology (i.e., in the sense of currents). From [2] this means that there exists $\Omega: I \rightarrow I_{3}(M)$, continuous in the mass norm, such that $\Omega(0)=0$ and $\partial \Omega(t)=\Sigma(t)$ for each $t \in[0,1]$. If we think of $\Omega(t)$ as domains of $M$, continuity in the mass norm means that

$$
\lim _{t \rightarrow t_{0}} \operatorname{vol}\left(\Omega(t) \Delta \Omega\left(t_{0}\right)\right)=0
$$

for every $t_{0} \in[0,1]$. Recall that $X \Delta Y=(X \backslash Y) \cup(Y \backslash X)$ denotes the symmetric difference between the sets $X$ and $Y$.

Since $\partial \Omega(1)=0$, we must have $\Omega(1)=k \cdot M$ for some $k \in \mathbb{Z}$ by the Constancy Theorem [27]. The sweepout $\Phi: I \rightarrow \mathcal{Z}_{2}(M)$ is said to be nontrivial if $k \neq 0$. The fact that the sweepouts used in the min-max procedure are nontrivial is important to guarantee that $W\left(\Pi_{1}\right)>0$.

The next theorem follows from the previous discussion: 
1.3. $4 \pi$ Theorem. Let $\Phi: I \rightarrow \mathcal{Z}_{2}\left(S^{3}\right)$ be a nontrivial sweepout of $S^{3}$. Then there exists $y \in[0,1]$ such that

$$
\operatorname{area}(\Phi(y)) \geq 4 \pi
$$

There are infinitely many closed minimal surfaces in $S^{3}$ (Lawson [14]). Among those, the simplest one after the equator is the Clifford torus

$$
\hat{\Sigma}=S^{1}\left(\frac{1}{\sqrt{2}}\right) \times S^{1}\left(\frac{1}{\sqrt{2}}\right) .
$$

This minimal surface has area $2 \pi^{2}$, index 5 , and, in fact, can be characterized by its index.

1.4. Theorem. (Urbano' 90 [30]) Let $\Sigma \subset S^{3}$ be a smooth, closed minimal surface with index $(\Sigma) \leq 5$. Then $\Sigma$ is either a great sphere or the Clifford torus, up to ambient isometries.

We have seen that the great sphere can appear as a minmax minimal surface. The question we posed ourselves, and that it turned out to be key to the solution of well-known global problems in conformal geometry such as the Willmore conjecture, was the following:

Question: Is it possible to produce the Clifford torus by min-max methods?

We have answered this question affirmatively by finding a natural class of five-parameter families of surfaces in $S^{3}$. Very briefly, to each embedded closed surface $\Sigma$ in $S^{3}$ of genus $g$, we associate a specific continuous 5 -parameter family of surfaces in $S^{3}$. (See Section 3 for details). Every surface in the family is the equidistant surface of some conformal image of $\Sigma$. This family is parametrized by a map $\Phi$ defined on the 5 -cube $I^{5}$, and is constructed so that

(1) $\Phi(x, 0)=\Phi(x, 1)=0$ (trivial surface) for any $x \in I^{4}$,

(2) $\{\Phi(x, t)\}_{t \in[0,1]}$ is the standard sweepout of $S^{3}$ by oriented round spheres centered at $Q(x) \in S^{3}$, for any $x \in \partial I^{4}$,

(3) $\Phi(x, 1 / 2)=\partial B_{\pi / 2}(Q(x))$, for any $x \in \partial I^{4}$,

(4) there is no concentration of area:

$$
\lim _{r \rightarrow 0} \sup \left\{\operatorname{area}\left(\Phi(x) \cap B_{r}(p)\right): p \in S^{3}, x \in I^{5}\right\}=0 .
$$

The main topological ingredient comes from the discovery that the degree of the center map $Q: \partial I^{4} \rightarrow S^{3}$ is equal to the genus $g$ of $\Sigma$. Therefore, if $g \geq 1$, this map $\Phi$ has the crucial property that its restriction to $\partial I^{4} \times\{1 / 2\}$ is a homotopically nontrivial map into the space of oriented great spheres, which is homeomorphic to $S^{3}$.

The min-max theory developed in [19] implies: 
1.5. $2 \pi^{2}$ Theorem. ([19]) Let $\Phi: I^{5} \rightarrow \mathcal{Z}_{2}\left(S^{3}\right)$ be a continuous map in the flat topology satisfying the properties (1)-(4) above, with center map $Q: \partial I^{4} \rightarrow S^{3}$. If $\operatorname{deg}(Q) \neq 0$, then there must exist $y \in I^{5}$ with

$$
\operatorname{area}(\Phi(y)) \geq 2 \pi^{2} \text {. }
$$

Let us now describe the applications to conformal geometry, the first being a solution to the Willmore conjecture. The Willmore energy of a closed surface $\Sigma$ immersed in Euclidean three-space is the total integral of the square of the mean curvature:

$$
\mathcal{W}(\Sigma)=\int_{\Sigma} H^{2} d \Sigma
$$

It was already known to Blaschke [6] and Thomsen [29] in the 1920s that this energy is conformally invariant, i.e., $\mathcal{W}(F(\Sigma))=\mathcal{W}(\Sigma)$ for any $F \in$ $\operatorname{Conf}\left(\mathbb{R}^{3}\right)$. The Willmore energy also appears naturally in some physical contexts as the bending energy of elastic membranes ([22], [10], [12]).

In the 1960s, Willmore proved the following result:

1.6. Theorem. (Willmore) Let $\Sigma$ be a smooth closed surface in $\mathbb{R}^{3}$. Then $\mathcal{W}(\Sigma) \geq 4 \pi$, and equality holds if and only if $\Sigma$ is a round sphere.

Hence in some sense the Willmore energy measures the deviation of a given surface from being round. It is then natural to ask what is the optimal shape among all surfaces of some fixed topological type. Motivated by the analysis of circular tori of revolution, Willmore made a conjecture for the case of genus one:

1.7. Willmore Conjecture $\mathbf{( 1 9 6 5 ,}[31])$. The integral of the square of the mean curvature of a torus immersed in $\mathbb{R}^{3}$ is at least $2 \pi^{2}$.

The equality is achieved by the torus of revolution whose generating circle has radius 1 and center at distance $\sqrt{2}$ from the axis of revolution:

$$
(u, v) \mapsto((\sqrt{2}+\cos u) \cos v,(\sqrt{2}+\cos u) \sin v, \sin u) \in \mathbb{R}^{3} .
$$

This torus can also be seen as a stereographic projection of the Clifford torus $S^{1}\left(\frac{1}{\sqrt{2}}\right) \times S^{1}\left(\frac{1}{\sqrt{2}}\right) \subset S^{3}$. In fact, since a stereographic projection $\pi: S^{3} \backslash$ $\{p\} \rightarrow \mathbb{R}^{3}$ is a conformal map, the conjecture can be formulated equivalently for surfaces in the three-sphere. Given $\Sigma \subset S^{3} \backslash\{p\}$, we have

$$
\int_{\tilde{\Sigma}} \tilde{H}^{2} d \tilde{\Sigma}=\int_{\Sigma}\left(1+H^{2}\right) d \Sigma
$$

where $H$ and $\tilde{H}$ are the mean curvature functions of $\Sigma \subset S^{3}$ and $\tilde{\Sigma}=\pi(\Sigma) \subset$ $\mathbb{R}^{3}$, respectively.

Therefore we make the following definition: 
1.8. Definition. The Willmore energy of a closed surface $\Sigma \subset S^{3}$ is the quantity:

$$
\mathcal{W}(\Sigma)=\int_{\Sigma}\left(1+H^{2}\right) d \Sigma
$$

Here $H$ denotes the mean curvature of $\Sigma$, i.e., $H=\frac{k_{1}+k_{2}}{2}$ where $k_{1}$ and $k_{2}$ are the principal curvatures.

The conjecture had been verified in many special cases. We refer the reader to [19] for the long history of partial results. In particular, it was proven by Li and Yau [15] through conformal invariance that the Willmore energy of any non-embedded surface must be at least $8 \pi$ (which is strictly bigger than $2 \pi^{2}$ ).

The Willmore conjecture then follows as a consequence of the next theorem, proven by the present authors in [19]:

Theorem 1. ([19]) Let $\Sigma \subset S^{3}$ be an embedded, smooth closed surface of genus $g \geq 1$. Then

$$
\mathcal{W}(\Sigma) \geq 2 \pi^{2}
$$

and the equality holds if and only if $\Sigma$ is the Clifford torus up to conformal transformations of $S^{3}$.

The proof of Theorem 1 is based on the construction, for each embedded closed surface $\Sigma$ of genus $g \geq 1$ in $\mathbb{R}^{3}$, of a suitable family $\Phi: I^{5} \rightarrow \mathcal{Z}_{2}\left(S^{3}\right)$ that satisfies the assumptions of the $2 \pi^{2}$ Theorem with $\operatorname{deg}(\mathrm{Q})=g$ and so area $(\Phi(y)) \geq 2 \pi^{2}$ for some $y \in I^{5}$. The family is constructed so that area $(\Phi(x)) \leq \mathcal{W}(\Sigma)$ for each $x \in I^{5}$ and this implies the theorem.

The second application comes from the theory of links in $\mathbb{R}^{3}$. Let $\gamma_{i}$ : $S^{1} \rightarrow \mathbb{R}^{3}, i=1,2$, be a 2-component link, i.e., a pair of closed curves in Euclidean three-space with $\gamma_{1}\left(S^{1}\right) \cap \gamma_{2}\left(S^{1}\right)=\emptyset$. The Möbius cross energy of the link $\left(\gamma_{1}, \gamma_{2}\right)$ is defined by

$$
E\left(\gamma_{1}, \gamma_{2}\right)=\int_{S^{1} \times S^{1}} \frac{\left|\gamma_{1}^{\prime}(s)\right|\left|\gamma_{2}^{\prime}(t)\right|}{\left|\gamma_{1}(s)-\gamma_{2}(t)\right|^{2}} d s d t .
$$

Like the Willmore energy, the Möbius energy has the remarkable property of being invariant under conformal transformations of $\mathbb{R}^{3}[9]$. In the case of knots other energies were considered by O'Hara [23].

Because of Gauss formula for the linking number $\operatorname{lk}\left(\gamma_{1}, \gamma_{2}\right)$ :

$$
\operatorname{lk}\left(\gamma_{1}, \gamma_{2}\right)=\frac{1}{4 \pi} \int_{S^{1} \times S^{1}} \frac{\operatorname{det}\left(\gamma_{1}^{\prime}(s), \gamma_{2}^{\prime}(t), \gamma_{1}(s)-\gamma_{2}(t)\right)}{\left|\gamma_{1}(s)-\gamma_{2}(t)\right|^{3}} d s d t
$$

we immediately get that $E\left(\gamma_{1}, \gamma_{2}\right) \geq 4 \pi\left|\operatorname{lk}\left(\gamma_{1}, \gamma_{2}\right)\right|$. It is natural to search for optimal configurations, i.e., minimizers of the Möbius energy.

It was conjectured by Freedman, He and Wang [9], in 1994, that the Möbius energy should be minimized, among the class of all nontrivial links in 
$\mathbb{R}^{3}$, by the stereographic projection of the standard Hopf link. The standard Hopf link $\left(\hat{\gamma}_{1}, \hat{\gamma}_{2}\right)$ is described by

$$
\hat{\gamma}_{1}(s)=(\cos s, \sin s, 0,0) \in S^{3} \quad \text { and } \quad \hat{\gamma}_{2}(t)=(0,0, \cos t, \sin t) \in S^{3},
$$

and it is simple to check that $E\left(\hat{\gamma}_{1}, \hat{\gamma}_{2}\right)=2 \pi^{2}$. Note that the definition of the energy and its conformal invariance property extend to any 2 -component link in $\mathbb{R}^{n}$ [13]. It follows from a result of He [11] that it suffices to prove the conjecture for links $\left(\gamma_{1}, \gamma_{2}\right)$ with linking number $\operatorname{lk}\left(\gamma_{1}, \gamma_{2}\right)= \pm 1$. This is what I. Agol and the present authors prove in [1]:

Theorem 2. ([1]) Let $\gamma_{i}: S^{1} \rightarrow \mathbb{R}^{3}, i=1,2$, be a 2-component link in $\mathbb{R}^{3}$ with $\left|\operatorname{lk}\left(\gamma_{1}, \gamma_{2}\right)\right|=1$. Then $E\left(\gamma_{1}, \gamma_{2}\right) \geq 2 \pi^{2}$.

Moreover, if $E\left(\gamma_{1}, \gamma_{2}\right)=2 \pi^{2}$ then there exists a conformal map $F: \mathbb{R}^{4} \rightarrow$ $\mathbb{R}^{4}$ such that $\left(F \circ \gamma_{1}, F \circ \gamma_{2}\right)$ describes the standard Hopf link up to orientation.

The proof of Theorem 2 is based on the construction, for each link in $\mathbb{R}^{3}$ with $\left|\operatorname{lk}\left(\gamma_{1}, \gamma_{2}\right)\right|=1$, of a suitable family $\Phi: I^{5} \rightarrow \mathcal{Z}_{2}\left(S^{3}\right)$ that satisfies the assumptions of the $2 \pi^{2}$ Theorem with $\operatorname{deg}(\mathrm{Q})= \pm 1$ and so area $(\Phi(y)) \geq 2 \pi^{2}$ for some $y \in I^{5}$. The family is constructed so that area $(\Phi(x)) \leq E\left(\gamma_{1}, \gamma_{2}\right)$ for each $x \in I^{5}$ and this implies the theorem.

This paper is organized as follows. In Section 2 we discuss the proof of the $2 \pi^{2}$ Theorem by min-max methods. In Section 3 we describe the 5 parameter family of surfaces in $S^{3}$ associated with the Willmore problem. In Section 4 we describe the 5-parameter family of surfaces in $S^{3}$ associated with the Möbius problem.

\section{The $2 \pi^{2}$ TheOREM}

Let $\left(M^{3}, g\right)$ be a compact Riemannian manifold. Let $I^{n}=[0,1]^{n}$, and suppose we have a continuous map $\Phi$ defined on $I^{n}$ such that $\Phi(x)$ is a compact surface with no boundary in $M$ for each $x \in I^{n}$. More precisely, let $\Phi: I^{n} \rightarrow \mathcal{Z}_{2}\left(M^{3}\right)$ be a map continuous in the flat topology. We denote by $\Pi$ the set of all maps $\Phi^{\prime}: I^{n} \rightarrow \mathcal{Z}_{2}\left(M^{3}\right)$ that are homotopic to $\Phi$ relative to the boundary $\partial I^{n}$. This means that there exists a homotopy $\Psi: I^{n+1} \rightarrow$ $\mathcal{Z}_{2}\left(M^{3}\right)$ such that:

- $\Psi$ is continuous in the flat topology;

- $\Psi(0, x)=\Phi(x)$ and $\Psi(1, x)=\Phi^{\prime}(x)$ for each $x \in I^{n}$;

- $\Psi(t, x)=\Phi(x)=\Phi^{\prime}(x)$ for every $t \in I, x \in \partial I^{n}$.

The width of $\Pi$ is then defined to be the min-max invariant:

$$
\mathbf{L}(\Pi)=\inf _{\Phi^{\prime} \in \Pi} \sup _{x \in I^{n}} \operatorname{area}\left(\Phi^{\prime}(x)\right) .
$$

We also say that a sequence $\left\{\Phi_{i}\right\}$ of maps in $\Pi$ is an optimal sequence if

$$
\lim _{i \rightarrow \infty} \sup _{x \in I^{n}} \operatorname{area}\left(\Phi_{i}(x)\right)=\mathbf{L}(\Pi) \text {. }
$$

The main goal of the Almgren-Pitts min-max theory is to realize the width as the area of a minimal surface. The prototypical theorem is: 
Min-Max Theorem. If

$$
\mathbf{L}(\Pi)>\sup _{x \in \partial I^{n}} \operatorname{area}(\Phi(x)),
$$

then there exists a smooth embedded closed minimal surface $\Sigma \subset M$ (possibly disconnected, with multiplicities) such that

$$
\operatorname{area}(\Sigma)=L(\Pi) \text {. }
$$

Moreover, if $\left\{\Phi_{i}\right\}$ is an optimal sequence then, after passing to a subsequence $\left\{\Phi_{j}\right\}$, we can choose $\Sigma$ to be the varifold limit, as $j \rightarrow \infty$, of $\Phi_{j}\left(x_{j}\right)$ for some $x_{j} \in I^{n}$. The mass functional is not continuous in the flat topology.

This is because integral currents are oriented objects subject to the wellknown phenomenon of cancellation of mass. Therefore it is natural to require stronger continuity assumptions on the map $\Phi: I^{n} \rightarrow \mathcal{Z}_{2}\left(M^{3}\right)$. For instance, we can ask that $\Phi$ be also continuous in varifold sense or even continuous with respect to the mass norm. On the other hand, the families that are important in the applications are only continuous in the flat topology. These issues are dealt with by approximation arguments (see Subsection 2.2 for some comments about that).

2.1. $2 \pi^{2}$ Theorem. ([19]) Let $\Phi: I^{5} \rightarrow \mathcal{Z}_{2}\left(S^{3}\right)$ be a continuous map in the flat topology satisfying the properties:

(1) $\Phi(x, 0)=\Phi(x, 1)=0$ for any $x \in I^{4}$,

(2) $\{\Phi(x, t)\}_{t \in[0,1]}$ is the standard sweepout of $S^{3}$ by oriented round spheres centered at $Q(x) \in S^{3}$, for any $x \in \partial I^{4}$,

(3) $\Phi(x, 1 / 2)=\partial B_{\pi / 2}(Q(x))$, for any $x \in \partial I^{4}$,

(4) there is no concentration of area:

$$
\lim _{r \rightarrow 0} \sup \left\{\operatorname{area}\left(\Phi(x) \cap B_{r}(p)\right): p \in S^{3}, x \in I^{5}\right\}=0 .
$$

If $\operatorname{deg}(Q) \neq 0$, then there must exist $y \in I^{5}$ with

$$
\operatorname{area}(\Phi(y)) \geq 2 \pi^{2} \text {. }
$$

Sketch of proof. The condition (4) is technical and it is used in [19] only to guarantee that we can pass to the discrete setting of Almgren and Pitts (see Subsection 2.2).

Let $\Pi$ be the homotopy class of $\Phi$ with fixed boundary values. The theorem is proved once we establish that $L(\Pi) \geq 2 \pi^{2}$.

The first important step is to prove that $L(\Phi)>4 \pi$. Suppose this is false. Then $L(\Pi)=4 \pi$, and there exists a sequence of maps $\left\{\phi_{i}\right\}_{i \in \mathbb{N}}$ in $\Pi$ (hence $\phi_{i}=\Phi$ on $\left.\partial I^{5}\right)$ such that

$$
\sup _{x \in I^{5}} \operatorname{area}\left(\phi_{i}(x)\right) \leq 4 \pi+\frac{1}{i} .
$$


Note that, for any sequence of paths

$$
\gamma_{i}:[0,1] \rightarrow I^{5} \quad \text { with } \quad \gamma_{i}(0) \in I^{4} \times\{0\}, \quad \gamma_{i}(1) \in I^{4} \times\{1\},
$$

the sweepouts $\left\{\phi_{i} \circ \gamma_{i}\right\}$ of $S^{3}$ form an optimal sequence for the one-dimensional homotopy class $\Pi_{1}$. The Min-max Theorem applied to $\Pi_{1}$ implies that, after passing to a subsequence $\left\{\phi_{j} \circ \gamma_{j}\right\}$, we can find $t_{j} \in I$ such that the surfaces $\phi_{j}\left(\gamma_{j}\left(t_{j}\right)\right)$ converge to some great sphere.

We denote by $\mathcal{T}$ the space of great spheres. Then we look at the set $\bar{A}(i)$ of all $x \in I^{5}$ such that the distance of the surface $\phi_{i}(x)$ to $\mathcal{T}$ (in varifold sense) is at least $\varepsilon$, where $\varepsilon>0$ is small to be chosen later. We define $A(i)$ to be the connected component of $\bar{A}(i)$ that contains $I^{4} \times\{0\}$.

We claim that the 5-dimensional region $A(i)$ does not intersect $I^{4} \times\{1\}$ if $i$ is sufficiently large. If this were false, we could find, after passing to a subsequence, a sequence of continuous paths $\gamma_{j}$ connecting the bottom to the top of the cube with $\gamma_{j}(I) \subset A(j)$. This is not possible because by definition no surface in $A(j)$ is ever too close to a great sphere in $\mathcal{T}$.

Let $R(i)$ be the closure of $\partial A(i) \cap \operatorname{int}\left(I^{5}\right)$, and let $C(i)=\partial A(i) \cap\left(\partial I^{4} \times I\right)$. Since $\partial A(i)$ has no boundary, we get that

$$
\partial C(i)=\partial R(i) \cup \partial\left(I^{4} \times\{0\}\right) .
$$

Therefore we have that $\partial R(i)$ is homologous to $\partial I^{4} \times\{0\}$ in $\partial I^{4} \times I$. Consequently, $\partial R(i)$ is also homologous to $\partial I^{4} \times\{1 / 2\}$ in $\partial I^{4} \times I$.

We have accomplished the construction, for sufficiently large $i$, of a 4dimensional submanifold $R(i) \subset I^{5}$ with the properties:

- $R(i)$ separates $I^{4} \times\{0\}$ from $I^{4} \times\{1\}$,

- $\partial R(i) \subset \partial I^{4} \times I$,

- $\partial R(i)$ is homologous to $\partial I^{4} \times\{1 / 2\}$ in $\partial I^{4} \times I$,

- for every $x \in R(i)$, the surface $\phi_{i}(x)$ is $\varepsilon$-close, in varifold sense, to some great sphere in $\mathcal{T}$.

Note that the second and fourth properties also imply that every point in $\partial R(i)$ is very close to $\partial I^{4} \times\{1 / 2\}$.

If $\varepsilon$ is chosen sufficiently small, and if we forget the orientations, we can approximate the restriction of $\phi_{i}$ to $R(i)$ by a continuous function

$$
f_{i}: R(i) \rightarrow \mathcal{T} \quad \text { such that } f_{i}(x, t)=\Phi(x, 1 / 2) \quad \text { for } \quad(x, t) \in \partial R(i) .
$$

Now let $\hat{\Phi}: \partial I^{4} \times I \rightarrow \mathcal{T}$ be given by $\hat{\Phi}(x, t)=\Phi(x, 1 / 2) \in \mathcal{T}$. Then, in homology,

$$
\hat{\Phi}_{*}[\partial R(i)]=f_{i_{*}}[\partial R(i)]=\left[f_{i \#} \partial(R(i))\right]=\left[\partial f_{i \#}(R(i))\right]=0 .
$$

On the other hand, since $\partial R(i)$ is homologous to $\partial I^{4} \times\{1 / 2\}$ in $\partial I^{4} \times I$,

$\hat{\Phi}_{*}[\partial R(i)]=\hat{\Phi}_{*}\left[\partial I^{4} \times\{1 / 2\}\right]=\Phi_{*}\left(\left[\partial I^{4} \times\{1 / 2\}\right]\right)=2 \operatorname{deg}(Q) \in H_{3}\left(\mathbb{R P}^{3}, \mathbb{Z}\right)$.

We have reached a contradiction if $\operatorname{deg}(Q) \neq 0$, hence $L(\Pi)>4 \pi$.

We claim that, in fact, $L(\Pi) \geq 2 \pi^{2}$. This is proven by looking at $\Sigma$, the minimal surface with least area among all minimal surfaces in $S^{3}$ with 
genus greater than or equal to 1. (The existence of $\Sigma$ follows from standard arguments in Geometric Measure Theory. This is explained in an appendix of [19].) The area of $\Sigma$ is of course bounded above by $2 \pi^{2}$, the area of the Clifford torus.

The idea is to argue that if index $(\Sigma) \geq 6$, then the 5-dimensional canonical family associated with $\Sigma$ (see Section 3 ) could be slightly perturbed so to produce a map $\Phi^{\prime}: I^{5} \rightarrow \mathcal{Z}_{2}\left(S^{3}\right)$ satisfying all assumptions of the $2 \pi^{2}$ Theorem plus

$$
\sup \left\{\operatorname{area}\left(\Phi^{\prime}(x)\right): x \in I^{5}\right\}<\operatorname{area}(\Sigma) .
$$

If $\Pi^{\prime}$ is the homotopy class of $\Phi^{\prime}$, what we have done so far proves that $\mathbf{L}\left(\Pi^{\prime}\right)>4 \pi$. Therefore we could apply the Min-Max Theorem to $\Pi^{\prime}$ in order to find an embedded minimal surface $\Sigma^{\prime}$ (with possible multiplicities) in $S^{3}$ such that

$$
\operatorname{area}\left(\Sigma^{\prime}\right)=\mathbf{L}\left(\Pi^{\prime}\right)>4 \pi
$$

But

$$
\mathbf{L}\left(\Pi^{\prime}\right) \leq \sup _{x \in I^{5}} \operatorname{area}\left(\Phi^{\prime}(x)\right)<\operatorname{area}(\Sigma) \leq 2 \pi^{2} .
$$

Thus area $\left(\Sigma^{\prime}\right)<\operatorname{area}(\Sigma) \leq 2 \pi^{2}$.

The area of any closed minimal surface in $S^{3}$ is at least $4 \pi$, hence the multiplicity of $\Sigma^{\prime}$ must be equal to one (otherwise area $\left(\Sigma^{\prime}\right) \geq 8 \pi$ ). Since, by Almgren [4], the only minimal surfaces of $S^{3}$ with genus zero are the great spheres, and area $\left(\Sigma^{\prime}\right)>4 \pi$, we get that $\operatorname{genus}\left(\Sigma^{\prime}\right) \geq 1$. This is in contradiction with the least-area property of $\Sigma$.

Therefore index $(\Sigma) \leq 5$. By Urbano's Theorem [30], $\Sigma$ is the Clifford torus up to isometries of $S^{3}$.

Now, by the Min-Max Theorem applied to $\Pi$, there exists an embedded minimal surface $\widehat{\Sigma}$ (with possible multiplicities) in $S^{3}$ such that

$$
\operatorname{area}(\widehat{\Sigma})=\mathbf{L}(\Pi) \text {. }
$$

Since we already know that $L(\Pi)>4 \pi$, either $\widehat{\Sigma}$ contains a component with multiplicity greater than 1 , in which case area $(\widehat{\Sigma}) \geq 8 \pi$, or it has nontrivial genus and then $\operatorname{area}(\widehat{\Sigma}) \geq \operatorname{area}(\Sigma)$. In any case we get

$$
\sup _{x \in I^{5}} \operatorname{area}(\Phi(x)) \geq L(\Pi)=\operatorname{area}(\widehat{\Sigma}) \geq 2 \pi^{2} .
$$

2.2. Technical comments. At this point it is worthwhile to make some technical comments. In reality, Almgren and Pitts work with a discretized version of the min-max theory described above: the maps are defined on the vertices of grids in $I^{n}$ that become finer and finer. The notion of continuity is replaced by the concept of fineness of a map, and appropriate discretized notions of homotopy have to be provided. Pitts chooses to work with families of currents that are fine in the mass norm $\mathbf{M}$. The advantage of using the M-norm in $\mathcal{Z}_{2}\left(M^{3}\right)$ is that it can easily be localized (unlike the $\mathbf{F}$-metric of 
varifold convergence), making it ideal for area comparisons, cut and paste arguments, and thus, regularity theory. The other advantage is that the mass functional is continuous in the $\mathbf{M}$-norm, as in the $\mathbf{F}$-metric (but not in the flat topology).

The disadvantage is that even the simplest family, like the 1-dimensional family $\left\{x_{4}=s\right\}$ in $S^{3}$, is not continuous with respect to the mass norm. This issue is addressed by discretizing the family $\left\{x_{4}=s\right\}$, and then interpolating, which means adding currents to the family or grid so that it becomes fine in the M-norm. The min-max procedure is then applied to the interpolated family.

In [19], we deal with these technical difficulties by following AlmgrenPitts approach. There are other treatments to min-max theory, such as $[28,7]$, but those require stronger regularity assumptions and do not apply to the families we are interested in. By discretizing and interpolating, we prove that we can replace a map $\Phi$ defined on $I^{n}$, continuous in the flat topology, by a sequence of discrete maps $\phi_{i}$ that are fine in the mass norm and approximate $\Phi$ in the flat topology. The interpolation is carried out in such a way that the supremum of $\mathbf{M}\left(\phi_{i}\right)$ is not much bigger than the supremum of $\mathbf{M}(\Phi)$.

\section{FAMily for the Willmore PROBlem}

Let $B^{4}$ be the unit ball. For every $v \in B^{4}$ we consider the conformal map

$$
F_{v}: S^{3} \rightarrow S^{3}, \quad F_{v}(x)=\frac{\left(1-|v|^{2}\right)}{|x-v|^{2}}(x-v)-v .
$$

Note that if $v \neq 0$ then $F_{v}$ is a centered dilation of $S^{3}$ that fixes $v /|v|$ and $-v /|v|$. To each smooth embedded closed surface $\Sigma \subset S^{3}$, we associate a canonical five-dimensional family of surfaces:

$$
\Sigma_{(v, t)}=\partial\left\{x \in S^{3}: d_{v}(x)<t\right\}, \quad(v, t) \in B^{4} \times[-\pi, \pi] .
$$

Here $d_{v}: S^{3} \rightarrow S^{3}$ denotes the signed distance function to the oriented surface $\Sigma_{v}=F_{v}(\Sigma)$, which becomes well defined after we choose a unit normal vector field $N$ to $\Sigma$ in $S^{3}$. The distance is computed with respect to the standard metric of $S^{3}$. Note that $\Sigma(v, \pi)=\Sigma(v,-\pi)=\emptyset$ for every $v \in B^{4}$.

The importance of this family is described in the next theorem. A related result appears in Proposition 1 of [24].

3.1. Theorem. We have, for every $(v, t) \in B^{4} \times(-\pi, \pi)$,

$$
\text { area }\left(\Sigma_{(v, t)}\right) \leq \mathcal{W}(\Sigma)
$$

Moreover, if $\Sigma$ is not a geodesic sphere and

$$
\text { area }\left(\Sigma_{(v, t)}\right)=\mathcal{W}(\Sigma)
$$

then $t=0$ and $\Sigma_{v}$ is a minimal surface. 
Proof. Let $N_{v}=D F_{v}(N) /\left|D F_{v}(N)\right|$ be the normal vector to $\Sigma_{v}=F_{v}(\Sigma)$ and consider the smooth map

$$
\psi_{(v, t)}: \Sigma_{v} \rightarrow S^{3}, \quad \psi_{(v, t)}(y)=\exp _{y}\left(t N_{v}(y)\right)=\cos t y+\sin t N_{v}(y) .
$$

A calculation gives $([24])$ :

$$
\operatorname{Jac} \psi_{(v, t)}(y)=\left(1+H(v)^{2}\right)-(\sin t+H(v) \cos t)^{2}-\frac{\left(k_{1}(v)-k_{2}(v)\right)^{2}}{4} \sin ^{2} t,
$$

where $k_{1}(v)$ and $k_{2}(v)$ are the principal curvatures of $\Sigma_{v}$ at $y$, and $H(v)=$ $\frac{k_{1}(v)+k_{2}(v)}{2}$ is the mean curvature.

Hence, by the area formula and the conformal invariance of the Willmore energy we obtain

$$
\begin{gathered}
\operatorname{area}\left(\Sigma_{(v, t)}\right) \leq \operatorname{area}\left(\psi_{(v, t)}\left(\left\{\operatorname{Jac} \psi_{(v, t)}(p) \geq 0\right\}\right)\right) \\
\leq \int_{\left\{\operatorname{Jac} \psi_{(v, t)} \geq 0\right\}}\left(\operatorname{Jac} \psi_{(v, t)}\right) d \Sigma_{v} \\
\leq \int_{\left\{\operatorname{Jac} \psi_{(v, t)} \geq 0\right\}}\left(1+H(v)^{2}\right)-\sin ^{2} t \frac{\left(k_{1}(v)-k_{2}(v)\right)^{2}}{4} d \Sigma_{v} \\
\leq \int_{\Sigma}\left(1+H(v)^{2}\right) d \Sigma_{v}=\mathcal{W}(\Sigma) .
\end{gathered}
$$

If equality holds for some $(v, t) \in B^{4} \times(-\pi, \pi)$, we obtain from the set of inequalities above that $\left\{\operatorname{Jac} \psi_{(v, t)} \geq 0\right\}=\Sigma$ and

$$
\frac{\sin ^{2} t}{2} \int_{\Sigma_{v}}|\AA|^{2} d \Sigma_{v}=\frac{\sin ^{2} t}{2} \int_{\Sigma}|\AA|^{2} d \Sigma=0,
$$

where $\AA$ denotes the trace-free part of the second fundamental form. This implies the rigidity statement.

We would like to apply the min-max method to families that are homotopic to the 5-dimensional family

$$
\left\{\Sigma_{(v, t)}\right\}_{(v, t) \in B^{4} \times[-\pi, \pi]},
$$

and therefore it is important to understand its behavior as

$$
(v, t) \rightarrow \partial\left(B^{4} \times[-\pi, \pi]\right)=S^{3} \times[-\pi, \pi] .
$$

We write $A_{(v, t)}=\left\{x \in S^{3}: d_{v}(x)<t\right\}$, so that $\partial A_{(v, t)}=\Sigma_{(v, t)}$, and take the convergence with respect to the flat topology: $\Sigma_{n}=\partial A_{n} \rightarrow T=\partial U$ if

$$
\lim _{n \rightarrow \infty} \operatorname{vol}\left(A_{n} \Delta U\right)=0 .
$$

In that sense, we can compute all possible limits. We let $A$ and $A^{*}$ denote the disjoint connected components of $S^{3} \backslash \Sigma=A \cup A^{*}$, chosen so that the unit normal $N$ to $\Sigma$ points into $A^{*}$. For $p \in S^{3}$ and $k \in[-\infty,+\infty]$, we set $\bar{Q}_{p, k}=-\frac{k}{\sqrt{1+k^{2}}} p-\frac{1}{\sqrt{1+k^{2}}} N(p) \in S^{3}$ and $\bar{r}_{k}=\frac{\pi}{2}-\arctan k \in[0, \pi]$. 
MIN-MAX THEORY, WILLMORE CONJECTURE AND THE ENERGY OF LINKS 13

3.2. Proposition. Consider a sequence $\left(v_{n}, t_{n}\right) \in B^{4} \times[-\pi, \pi]$ converging to $(v, t) \in \bar{B}^{4} \times[-\pi, \pi]$.

(i) If $v \in B^{4}$ then

$$
\lim _{n \rightarrow \infty} \operatorname{vol}\left(A_{\left(v_{n}, t_{n}\right)} \Delta A_{(v, t)}\right)=0 .
$$

(ii) If $v \in A$ then

$$
\lim _{n \rightarrow \infty} \operatorname{vol}\left(A_{\left(v_{n}, t_{n}\right)} \Delta B_{\pi+t}(v)\right)=0 .
$$

(iii) If $v \in A^{*}$ then

$$
\lim _{n \rightarrow \infty} \operatorname{vol}\left(A_{\left(v_{n}, t_{n}\right)} \Delta B_{t}(-v)\right)=0 .
$$

(iv) If $v=p \in \Sigma$ and

$$
v_{n}=\left(1-s_{n 1}\right)\left(\cos \left(s_{n 2}\right) p_{n}+\sin \left(s_{n 2}\right) N\left(p_{n}\right)\right), \quad \lim _{n \rightarrow \infty} \frac{s_{n 2}}{s_{n 1}}=k \in[-\infty,+\infty],
$$

then

$$
\lim _{n \rightarrow \infty} \operatorname{vol}\left(A_{\left(v_{n}, t_{n}\right)} \Delta B_{\bar{r}_{k}+t}\left(\bar{Q}_{p, k}\right)\right)=0 .
$$

The proof of this proposition ([19]) follows from a careful analysis of the effect of the conformal transformations $F_{v}: S^{3} \rightarrow S^{3}$ on the surface $\Sigma$. See that, as $v \in B^{4}$ converges to $p \in \Sigma$, the geodesic sphere we get in the limit will depend on the angle of convergence. The idea now is to fix this failure of continuity by reparametrizing the canonical family. This is done by "blowing-up" $\bar{B}^{4}$ along the surface $\Sigma$, a procedure which we describe now.

Let $\Omega_{\varepsilon}$ be a tubular neighborhood of radius $\varepsilon$ (chosen to be small) around $\Sigma$ in $\bar{B}^{4}$ :

$$
\Omega_{\varepsilon}=\left\{\left(1-s_{1}\right)\left(\cos \left(s_{2}\right) p+\sin \left(s_{2}\right) N(p)\right):\left|\left(s_{1}, s_{2}\right)\right|<\varepsilon, s_{1} \geq 0\right\} .
$$

We take a continuous map $T: \bar{B}^{4} \rightarrow \bar{B}^{4}$ such that:

- $T$ maps $B^{4} \backslash \bar{\Omega}_{\varepsilon}$ homeomorphically onto $B^{4}$;

- $T$ maps $\bar{\Omega}_{\varepsilon}$ onto $\Sigma$ by nearest point projection;

- the map

$$
C(v, t)=\Sigma_{(T(v), t)}, \quad(v, t) \in\left(B^{4} \backslash \bar{\Omega}_{\varepsilon}\right) \times[-\pi, \pi],
$$

admits a continuous extension to $\overline{\left(B^{4} \backslash \Omega_{\varepsilon}\right)} \times[-\pi, \pi]$, which we still denote by $C$.

Finally we extend $C$ to $\Omega_{\varepsilon}$ so that $C$ is constant along the $s_{1}$ direction inside $\Omega_{\varepsilon}$. The resulting map $C$, defined on $\bar{B}^{4} \times[-\pi, \pi]$, satisfies the following properties:

(i) $\operatorname{area}(C(v, \pi))=\operatorname{area}(C(v,-\pi))=0$ for every $v \in \bar{B}^{4}$;

(ii) $C(v, t)$ is a geodesic sphere whenever $v \in S^{3} \cup \bar{\Omega}_{\varepsilon}$; 
(iii) for each $v \in S^{3}$, there exists a unique $s(v) \in[-\pi / 2, \pi / 2]$ such that $C(v, s(v))$ is a great sphere, i.e., such that

$$
C(v, s(v))=\partial B_{\pi / 2}(\bar{Q}(v))
$$

for some $\bar{Q}(v) \in S^{3}$.

If we take into account the orientation, $\partial B_{\pi / 2}(p) \neq \partial B_{\pi / 2}(-p)$. Hence $\bar{Q}(v)$ is also unique.

The main topological ingredient is the discovery that:

$$
\bar{Q}: S^{3} \rightarrow S^{3} \text { is a continuous map with degree equal to } g \text {. }
$$

This means that the canonical family detects the genus of $\Sigma$, and this is what will make the min-max approach work.

3.3. Lemma. The degree of $\bar{Q}: S^{3} \rightarrow S^{3}$ is equal to $g$.

Sketch. We will use the fact that $\bar{Q}$ is piecewise smooth. We have that $\bar{Q}=-T$ on $A^{*} \backslash \bar{\Omega}_{\varepsilon}$, that $\bar{Q}=T$ on $A \backslash \bar{\Omega}_{\varepsilon}$, and that

$$
\bar{Q}(v)=-\frac{t}{\varepsilon} p-\frac{\sqrt{\varepsilon^{2}-t^{2}}}{\varepsilon} N(p)
$$

for every $v=\cos t p+\sin t N(p) \in S^{3} \cap \bar{\Omega}_{\varepsilon}$. Let $d V$ denote the volume form of $S^{3}$ and $\nabla$ the induced connection on $S^{3}$.

Since $T$ is an orientation-preserving diffeomorphism of $A^{*} \backslash \Omega_{\varepsilon}$ onto $\overline{A^{*}}$, we get

$$
\int_{A^{*} \backslash \bar{\Omega}_{\varepsilon}} \bar{Q}^{*}(d V)=\int_{-A^{*}} d V=\operatorname{vol}\left(A^{*}\right)
$$

Similarly,

$$
\int_{A \backslash \bar{\Omega}_{\varepsilon}} \bar{Q}^{*}(d V)=\int_{A} d V=\operatorname{vol}(A)
$$

In order to compute the integral $\int_{S^{3} \cap \bar{\Omega}_{\varepsilon}} \bar{Q}^{*}(d V)$, we introduce the diffeomorphism $G: \Sigma \times[-\varepsilon, \varepsilon] \rightarrow S^{3} \cap \bar{\Omega}_{\varepsilon}$ defined by

$$
G(p, t)=\cos t p+\sin t N(p) \text {. }
$$

Recall that $\left\{e_{1}, e_{2}, e_{3}\right\} \in T_{p} S^{3}$ is a positive basis if $\left\{e_{1}, e_{2}, e_{3}, p\right\}$ is a positive basis of $\mathbb{R}^{4}$, and $\left\{e_{1}, e_{2}\right\} \in T_{p} \Sigma$ is a positive basis if $\left\{e_{1}, e_{2}, N(p)\right\}$ is a positive basis of $T_{p} S^{3}$. The orientation of $\Sigma \times[-\varepsilon, \varepsilon]$ is chosen so that $\left\{e_{1}, e_{2}, \partial_{t}\right\}$ is a positive basis whenever $\left\{e_{1}, e_{2}\right\}$ is a positive basis of $T \Sigma$. We have

$$
G_{*}\left(e_{1} \wedge e_{2} \wedge \partial_{t}\right)_{\mid(p, 0)}=e_{1} \wedge e_{2} \wedge N(p)
$$

and thus $G$ is orientation preserving.

Consider $Q=\bar{Q} \circ G: \Sigma \times[-\varepsilon, \varepsilon] \rightarrow S^{3}$. Then

$$
\int_{S^{3} \cap \bar{\Omega}_{\varepsilon}} \bar{Q}^{*}(d V)=\int_{\Sigma \times[-\varepsilon, \varepsilon]} G^{*}\left(\bar{Q}^{*}(d V)\right)=\int_{\Sigma \times[-\varepsilon, \varepsilon]} Q^{*}(d V) .
$$


But

$$
Q(p, t)=-\frac{t}{\varepsilon} p-\frac{\sqrt{\varepsilon^{2}-t^{2}}}{\varepsilon} N(p) .
$$

If $\left\{e_{1}, e_{2}\right\}$ is a positive orthonormal basis of $T_{p} \Sigma$ which diagonalizes the second fundamental form:

$$
\nabla_{e_{i}} N=-k_{i} e_{i} \quad \text { for } i=1,2,
$$

we can compute

$$
Q^{*}(d V)_{\mid(p, t)}\left(e_{1}, e_{2}, \partial_{t}\right)=\left(-\frac{t}{\varepsilon}+\frac{\sqrt{\varepsilon^{2}-t^{2}}}{\varepsilon} k_{1}\right)\left(-\frac{t}{\varepsilon}+\frac{\sqrt{\varepsilon^{2}-t^{2}}}{\varepsilon} k_{2}\right) \frac{(-1)}{\sqrt{\varepsilon^{2}-t^{2}}} .
$$

The Gauss equation implies that $K=1+k_{1} k_{2}$, where $K$ denotes the Gauss curvature of $\Sigma$ and so we conclude that

$$
\begin{aligned}
& \int_{\Sigma \times[-\varepsilon, \varepsilon]} Q^{*}(d V) \\
& =-\int_{\Sigma} \int_{-\varepsilon}^{\varepsilon} \frac{1}{\varepsilon^{2}}\left(k_{1} k_{2} \sqrt{\varepsilon^{2}-t^{2}}-\left(k_{1}+k_{2}\right) t+\frac{t^{2}}{\sqrt{\varepsilon^{2}-t^{2}}}\right) d t d \Sigma \\
& \quad=-\frac{\pi}{2} \int_{\Sigma}(K-1) d \Sigma-\frac{\pi}{2} \int_{\Sigma} d \Sigma=-\pi^{2} \chi(\Sigma)=\pi^{2}(2 g-2) .
\end{aligned}
$$

Finally, since $\operatorname{vol}\left(S^{3}\right)=2 \pi^{2}$ (an interesting fact!), we combine (2), (3), and (4) to obtain

$$
\begin{gathered}
\int_{S^{3}} \bar{Q}^{*}(d V)=\int_{A^{*} \backslash \bar{\Omega}_{\varepsilon}} \bar{Q}^{*}(d V)+\int_{A \backslash \bar{\Omega}_{\varepsilon}} \bar{Q}^{*}(d V)+\int_{S^{3} \cap \bar{\Omega}_{\varepsilon}} \bar{Q}^{*}(d V) \\
=\operatorname{vol}\left(A^{*}\right)+\operatorname{vol}(A)+\int_{\Sigma \times[-\varepsilon, \varepsilon]} Q^{*}(d V) \\
=2 \pi^{2}+\pi^{2}(2 g-2)=2 \pi^{2} g=g \cdot \int_{S^{3}} d V .
\end{gathered}
$$

It follows that $\operatorname{deg}(Q)=g$.

By choosing an orientation preserving homeomorphism $f: I^{4} \rightarrow \bar{B}^{4}$ (hence $f_{\mid \partial I^{4}}$ is a homeomorphism from $\partial I^{4}$ onto $S^{3}$ ), it is not difficult to reparametrize $C$ to get a map $\Phi$ defined on $I^{5}$ with the following properties:

3.4. Theorem. Let $\Sigma \subset S^{3}$ be an embedded closed surface of genus $g$. The map

$$
\Phi: I^{5} \rightarrow \mathcal{Z}_{2}\left(S^{3}\right)
$$

obtained by reparametrization of $C$, is continuous in the flat topology and satisfies:

(1) $\Phi(x, 0)=\Phi(x, 1)=0$ for any $x \in I^{4}$,

(2) $\{\Phi(x, t)\}_{t \in[0,1]}$ is the standard sweepout of $S^{3}$ by oriented round spheres centered at $Q(x) \in S^{3}$, for any $x \in \partial I^{4}$, 
(3) $\Phi(x, 1 / 2)=\partial B_{\pi / 2}(Q(x))$, for any $x \in \partial I^{4}$,

(4) there is no concentration of area:

$$
\lim _{r \rightarrow 0} \sup \left\{\operatorname{area}\left(\Phi(x) \cap B_{r}(p)\right): p \in S^{3}, x \in I^{5}\right\}=0,
$$

(5) $\sup \left\{\operatorname{area}(\Phi(x)): x \in I^{5}\right\} \leq \mathcal{W}(\Sigma)$,

(6) the center map $Q: \partial I^{4} \rightarrow S^{3}$ satisfies $\operatorname{deg}(Q)=g$.

Informally, the min-max family $\Phi$ can be thought of as an element of the relative homotopy group $\pi_{5}(\mathcal{S}, \mathcal{G})$, where $\mathcal{S}$ denotes the space of 2 -surfaces in $S^{3}$ as before and $\mathcal{G}$ denotes the space of geodesic spheres.

Given a smooth, embedded, closed surface $\Sigma \subset S^{3}$ with genus $g \geq 1$, it follows from the above properties that the map $\Phi: I^{5} \rightarrow \mathcal{Z}_{2}\left(S^{3}\right)$ satisfies all the assumptions of the $2 \pi^{2}$ Theorem. Therefore there exists $y \in I^{5}$ such that area $(\Phi(y)) \geq 2 \pi^{2}$. Since area $(\Phi(x)) \leq \mathcal{W}(\Sigma)$ for every $x \in I^{5}$, we get $\mathcal{W}(\Sigma) \geq 2 \pi^{2}$. In particular this proves the Willmore conjecture.

\section{FAmily fOR the MÖBIUS PROBlem}

Let $\gamma_{i}: S^{1} \rightarrow \mathbb{R}^{4}, i=1,2$, be a 2 -component link, i.e., a pair of rectifiable curves with $\gamma_{1}\left(S^{1}\right) \cap \gamma_{2}\left(S^{1}\right)=\emptyset$. After a reparametrization, we can assume $\gamma_{1}$ and $\gamma_{2}$ are Lipschitz and parametrized proportionally to arc length. The Gauss map of $\left(\gamma_{1}, \gamma_{2}\right)$ in $\mathbb{R}^{4}$, denoted by $g=G\left(\gamma_{1}, \gamma_{2}\right)$, is the Lipschitz map $g: S^{1} \times S^{1} \rightarrow S^{3}$ defined by

$$
g(s, t)=\frac{\gamma_{1}(s)-\gamma_{2}(t)}{\left|\gamma_{1}(s)-\gamma_{2}(t)\right|}
$$

Given $v \in \mathbb{R}^{4}$, we define the conformal map

$$
F_{v}: \mathbb{R}^{4} \backslash\{v\} \rightarrow \mathbb{R}^{4}, \quad F_{v}(x)=\frac{x-v}{|x-v|^{2}} .
$$

If $v \in B^{4}$, we have that

$$
F_{v}\left(S_{1}^{3}(0)\right)=S_{\frac{1}{1-|v|^{2}}}^{3}(c(v)) \quad \text { where } \quad c(v)=\frac{v}{1-|v|^{2}} .
$$

Given $w \in \mathbb{R}^{4}$ and $\lambda \in \mathbb{R}$, we set $D_{w, \lambda}(x)=\lambda(x-w)+w$, where $x \in \mathbb{R}^{4}$. Finally, given $v \in \bar{B}^{4}$ and $z \in(0,1)$, we also define

$$
b(v, z)=\frac{(2 z-1)}{\left(1-|v|^{2}+z\right)(1-z)}
$$

and

$$
a(v, z)=1+\left(1-|v|^{2}\right) b(v, z)=1+\frac{\left(1-|v|^{2}\right)(2 z-1)}{\left(1-|v|^{2}+z\right)(1-z)} .
$$

For each $v \in B^{4}$ fixed, $z \rightarrow a(v, z)$ is a nondecreasing parametrization of $(0,+\infty)$.

Suppose now that $\gamma_{1}\left(S^{1}\right) \cup \gamma_{2}\left(S^{1}\right) \subset S^{3}$. The canonical five-dimensional family of surfaces associated to $\left(\gamma_{1}, \gamma_{2}\right)$ is given by

$$
C(v, z)=g_{(v, z) \#}\left(S^{1} \times S^{1}\right) \in \mathcal{Z}_{2}\left(S^{3}\right),
$$


for $(v, z) \in B^{4} \times(0,1)$, where $g_{(v, z)}: S^{1} \times S^{1} \rightarrow S^{3}$ is defined by

$$
g_{(v, z)}(s, t)=\frac{\left(F_{v} \circ \gamma_{1}\right)(s)-\left(D_{c(v), a(v, z)} \circ F_{v} \circ \gamma_{2}\right)(t)}{\left|\left(F_{v} \circ \gamma_{1}\right)(s)-\left(D_{c(v), a(v, z)} \circ F_{v} \circ \gamma_{2}\right)(t)\right|} .
$$

Note that $g_{(v, z)}=G\left(F_{v} \circ \gamma_{1}, D_{c(v), a(v, z)} \circ F_{v} \circ \gamma_{2}\right)$.

Recall that, given a Lipschitz map $g: S^{1} \times S^{1} \rightarrow S^{3}$ (where $S^{1} \times S^{1}$ has a chosen orientation), the current $g_{\#}\left(S^{1} \times S^{1}\right) \in \mathcal{Z}_{2}\left(S^{3}\right)$ is defined by

$$
g_{\#}\left(S^{1} \times S^{1}\right)(\phi)=\int_{S^{1} \times S^{1}} g^{*} \phi, \quad \phi \in \mathcal{D}^{2}\left(\mathbb{R}^{4}\right) .
$$

We always have

$$
\mathbf{M}\left(g_{\#}\left(S^{1} \times S^{1}\right)\right) \leq \int_{S^{1} \times S^{1}}|\operatorname{Jac} g| d s d t .
$$

Here $\mathcal{D}^{2}\left(\mathbb{R}^{4}\right)$ denotes the space of smooth 2 -forms in $\mathbb{R}^{4}$ with compact support. The mass is defined by

$$
\mathbf{M}(T)=\sup \left\{T(\phi): \phi \in \mathcal{D}^{2}\left(\mathbb{R}^{4}\right),\|\phi\| \leq 1\right\}
$$

where $\|\phi\|$ denotes the comass norm of $\phi$.

Intuitively, $C(v, z)$ is the image of the Gauss map of the link obtained by applying the conformal transformation $F_{v}$ to $\left(\gamma_{1}, \gamma_{2}\right)$ and then dilating the curve $F_{v} \circ \gamma_{2}$ with respect to the center $c(v)$ by a factor of $a(v, z)$. Both curves $F_{v} \circ \gamma_{1}$ and $D_{c(v), a(v, z)} \circ F_{v} \circ \gamma_{2}$ are contained in spheres centered at $c(v)$. Notice that $g_{(v, 1 / 2)}=G\left(F_{v} \circ \gamma_{1}, F_{v} \circ \gamma_{2}\right)$, since $a(v, 1 / 2)=1$.

The following proposition is crucial:

4.1. Proposition. For every $(v, z) \in B^{4} \times(0,1)$, we have

$$
\operatorname{area}(C(v, z)) \leq E\left(\gamma_{1}, \gamma_{2}\right)
$$

Proof. The proposition follows from the estimates

$$
\begin{aligned}
\left|\operatorname{Jac} g_{(v, z)}\right|(s, t) \leq \frac{\left|\left(F_{v} \circ \gamma_{1}\right)^{\prime}(s)\right|\left|\left(D_{c(v), a(v, z)} \circ F_{v} \circ \gamma_{2}\right)^{\prime}(t)\right|}{\left|F_{v} \circ \gamma_{1}(s)-D_{c(v), a(v, z)} \circ F_{v} \circ \gamma_{2}(t)\right|^{2}} \\
\quad \leq \frac{a(v, z)\left|\left(F_{v} \circ \gamma_{1}\right)^{\prime}(s)\right|\left|\left(F_{v} \circ \gamma_{2}\right)^{\prime}(t)\right|}{a(v, z)\left|F_{v} \circ \gamma_{1}(s)-F_{v} \circ \gamma_{2}(t)\right|^{2}+b(v, z)^{2}}
\end{aligned}
$$

and the fact that $E\left(F_{v} \circ \gamma_{1}, F_{v} \circ \gamma_{2}\right)=E\left(\gamma_{1}, \gamma_{2}\right)$.

Like in the Willmore problem, it is important to determine the behavior of $C(v, z)$ as $(v, z) \rightarrow \partial\left(B^{4} \times(0,1)\right)$. Since the map $C$ turns out to be uniformly continuous in the flat topology, it admits an extension $C: \bar{B}^{4} \times$ $[0,1] \rightarrow \mathcal{Z}_{2}\left(S^{3}\right)$. We can then prove: 
4.2. Proposition. There exists a constant $c>0$ such that for every $p \in S^{3}$ we have

(i) $C(p, 1 / 2)=-\operatorname{lk}\left(\gamma_{1}, \gamma_{2}\right) \cdot \partial B_{\pi / 2}(p)$,

(ii) $\operatorname{supp}(C(p, z)) \subset \bar{B}_{\pi / 2}(p) \backslash B_{r(z)}(p)$ if $z \in[1 / 2,1]$,

(iii) $\operatorname{supp}(C(p, z)) \subset \bar{B}_{\pi / 2}(-p) \backslash B_{\pi-r(z)}(-p)$ if $z \in[0,1 / 2]$,

where

$$
r(z)=\cos ^{-1}\left(\frac{b(z)}{\sqrt{|b(z)|^{2}+c^{2}}}\right) \in[0, \pi] \quad \text { and } \quad b(z)=\frac{2 z-1}{z(1-z)} .
$$

Notice that $r(0)=\pi, r(1 / 2)=\pi / 2$, and $r(1)=0$.

Proof. Given $p \in S^{3} \backslash\left(\gamma_{1}\left(S^{1}\right) \cup \gamma_{2}\left(S^{1}\right)\right)$,

$$
\left(F_{p} \circ \gamma_{1}, F_{p} \circ \gamma_{2}\right)
$$

is a link in the affine hyperplane

$$
P_{(p, 1 / 2)}=\left\{x \in \mathbb{R}^{4}:\langle x, p\rangle=-1 / 2\right\},
$$

where $F_{p}$ sends the exterior unit normal of $S^{3}$ into $p$. This implies that

$$
\operatorname{supp} C(p, 1 / 2) \subset G\left(F_{p} \circ \gamma_{1}, F_{p} \circ \gamma_{2}\right)\left(S^{1} \times S^{1}\right) \subset \partial B_{\pi / 2}(-p),
$$

and so by the Constancy Theorem [27, Theorem 26.27] we have that

$$
C(p, 1 / 2)=k \cdot \partial B_{\pi / 2}(-p)
$$

for some integer $k$.

Let $P$ be the oriented hyperplane with normal vector $p \in S^{3}$ and let $\omega_{P}$ be its volume form. Let $\omega, \omega_{S^{3}}, \omega_{\mathbb{R}^{4}}$ denote, respectively, the volume form of $\partial B_{\pi / 2}(-p) \subset S^{3}$ (the exterior unit normal is $p$ ), $S^{3}$, and $\mathbb{R}^{4}$. We also have

$$
\partial B_{\pi / 2}(-p)=\left\{x \in \mathbb{R}^{4}:\langle x, p\rangle=0\right\} \cap S^{3} \subset P .
$$

If $g=G\left(\tilde{\gamma}_{1}, \tilde{\gamma}_{2}\right), \tilde{\gamma}_{i}=F_{p} \circ \gamma_{i}$, then

$$
\begin{aligned}
g^{*} \omega\left(\frac{\partial}{\partial s}, \frac{\partial}{\partial t}\right) & =\omega\left(\frac{\partial g}{\partial s}, \frac{\partial g}{\partial t}\right)=\omega_{S^{3}}\left(\frac{\partial g}{\partial s}, \frac{\partial g}{\partial t}, p\right) \\
& =\omega_{\mathbb{R}^{4}}\left(\frac{\partial g}{\partial s}, \frac{\partial g}{\partial t}, p, g\right)=-\omega_{\mathbb{R}^{4}}\left(\frac{\partial g}{\partial s}, \frac{\partial g}{\partial t}, g, p\right) \\
& =-\omega_{P}\left(\frac{\partial g}{\partial s}, \frac{\partial g}{\partial t}, g\right)=-\operatorname{det}\left(\frac{\partial g}{\partial s}, \frac{\partial g}{\partial t}, g\right) \\
& =\frac{\operatorname{det}\left(\tilde{\gamma}_{1}^{\prime}, \tilde{\gamma}_{2}^{\prime}, \tilde{\gamma}_{1}-\tilde{\gamma}_{2}\right)}{\left|\tilde{\gamma}_{1}-\tilde{\gamma}_{2}\right|^{3}}
\end{aligned}
$$

and so

$$
k=\frac{1}{4 \pi} C(p, 1 / 2)(\omega)=\frac{1}{4 \pi} \int_{S^{1} \times S^{1}} g^{*} \omega=\operatorname{lk}\left(\tilde{\gamma}_{1}, \tilde{\gamma}_{2}\right)=\operatorname{lk}\left(\gamma_{1}, \gamma_{2}\right) .
$$


By continuity of $C$, we also have $C(p, 1 / 2)=-\operatorname{lk}\left(\gamma_{1}, \gamma_{2}\right) \cdot \partial B_{\pi / 2}(p)$ for $p \in \gamma_{1}\left(S^{1}\right) \cup \gamma_{2}\left(S^{1}\right)$. This proves item (i).

If $z=0$ or $z=1$, we have $C(p, z)=0$ and the proposition follows immediately. Suppose $z \in(0,1)$ and $p \in S^{3} \backslash\left(\gamma_{1}\left(S^{1}\right) \cup \gamma_{2}\left(S^{1}\right)\right)$. Then

$$
\operatorname{supp}(C(p, z)) \subset g_{(p, z)}\left(S^{1} \times S^{1}\right) .
$$

If $v_{i} \in B^{4}$ converges to $p \in S^{3} \backslash\left(\gamma_{1}\left(S^{1}\right) \cup \gamma_{2}\left(S^{1}\right)\right)$, then

$$
\begin{aligned}
\lim _{i \rightarrow \infty} D_{c\left(v_{i}\right), a\left(v_{i}, z\right)} \circ F_{v_{i}}(x) & =\lim _{i \rightarrow \infty} a\left(v_{i}, z\right) F_{v_{i}}(x)-v_{i} \frac{a\left(v_{i}, z\right)-1}{1-\left|v_{i}\right|^{2}} \\
& =F_{p}(x)-b(z) p .
\end{aligned}
$$

Therefore we define the smooth map

$$
L_{(p, z)}: S^{3} \backslash\{p\} \rightarrow \mathbb{R}^{4}, \quad L_{(p, z)}(x)=F_{p}(x)-b(z) p,
$$

and note that

$$
\left\langle L_{(p, z)}(x), p\right\rangle=\left\langle\frac{x-p}{|x-p|^{2}}, p\right\rangle-b(z)=\frac{\langle x, p\rangle-1}{2-2\langle x, p\rangle}-b(z)=-1 / 2-b(z) .
$$

We have

$$
\begin{aligned}
\left\langle g_{(p, z)}, p\right\rangle & =\left\langle\frac{F_{p} \circ \gamma_{1}-L_{(p, z)} \circ \gamma_{2}}{\left|F_{p} \circ \gamma_{1}-L_{(p, z)} \circ \gamma_{2}\right|}, p\right\rangle \\
& =\left\langle\frac{F_{p} \circ \gamma_{1}+\frac{1}{2} p-\left(L_{(p, z)} \circ \gamma_{2}+\frac{1}{2} p\right)}{\left|F_{p} \circ \gamma_{1}-L_{(p, z)} \circ F_{p} \circ \gamma_{2}\right|}, p\right\rangle \\
& =\frac{b(p, z)}{\left|F_{p} \circ \gamma_{1}-L_{(p, z)} \circ F_{p} \circ \gamma_{2}\right|} .
\end{aligned}
$$

Hence $\left\langle g_{(p, z)}, p\right\rangle \geq 0$ if $z \in[1 / 2,1]$, and $\left\langle g_{(p, z)}, p\right\rangle \leq 0$ if $z \in[0,1 / 2]$.

Using the fact that for some constant $c>0$ we have

$$
\left|F_{p} \circ \gamma_{1}(s)-F_{p} \circ \gamma_{2}(t)\right|^{2} \geq c^{2}
$$

for all $p \in S^{3}$ and $(s, t) \in S^{1} \times S^{1}$, it follows that

$$
\left|\left\langle g_{(p, z)}(s, t), p\right\rangle\right| \leq \frac{|b(z)|}{\sqrt{c^{2}+b(z)^{2}}} .
$$

This proves items (ii) and (iii) of the proposition for $z \in[0,1]$ and $p \in$ $S^{3} \backslash\left(\gamma_{1}\left(S^{1}\right) \cup \gamma_{2}\left(S^{1}\right)\right)$. Since $S^{3} \backslash\left(\gamma_{1}\left(S^{1}\right) \cup \gamma_{2}\left(S^{1}\right)\right)$ is everywhere dense in $S^{3}$, and $C: S^{3} \times[0,1] \rightarrow \mathcal{Z}_{2}\left(S^{3}\right)$ is continuous in the flat topology, the proposition also holds for $p \in \gamma_{1}\left(S^{1}\right) \cup \gamma_{2}\left(S^{1}\right)$.

Note that the boundary values of $C$ are not necessarily round spheres, so we cannot yet apply the $2 \pi^{2}$ Theorem. As we explain next, the information given by the previous proposition is enough to find an extension of $C$ having that property. 
Let $p \in S^{3}, \lambda \in[0, \pi / 2]$. For $t \in[0,1]$, we define a retraction map

$$
R_{(p, \lambda, t)}: \bar{B}_{\pi / 2}(p) \backslash B_{\lambda}(p) \rightarrow \bar{B}_{\pi / 2}(p) \backslash B_{\lambda}(p)
$$

by

$$
R_{(p, \lambda, t)}(x)=\exp _{p}\left(\left((1-t)+t \frac{\lambda}{d(p, x)}\right) \exp _{p}^{-1}(x)\right) .
$$

Notice that $R_{(p, \pi / 2, t)}: \partial B_{\pi / 2}(p) \rightarrow \partial B_{\pi / 2}(p)$ is the identity map for every $p \in S^{3}$ and $t \in[0,1]$. The $R_{(p, \lambda, t)}$ are length-decreasing maps that satisfy

- $R_{(p, \lambda, 0)}(x)=x$ for all $x \in \bar{B}_{\pi / 2}(p) \backslash B_{\lambda}(p)$,

- $R_{(p, \lambda, 1)}\left(\bar{B}_{\pi / 2}(p) \backslash B_{\lambda}(p)\right) \subset \partial B_{\lambda}(p)$.

We now define $\tilde{C}: \bar{B}_{2}^{4}(0) \times[0,1] \rightarrow \mathcal{Z}_{2}\left(S^{3}\right)$ by

$$
\begin{aligned}
& \tilde{C}(v, t) \\
& =\left\{\begin{array}{lc}
C(v, t) & \text { if } v \in \bar{B}_{1}^{4}(0) \\
R\left(-\frac{v}{|v|}, \pi-r(t),|v|-1\right)_{\#} C\left(\frac{v}{|v|}, t\right) & \text { and } t \in[0,1], \\
R\left(\frac{v}{|v|}, r(t),|v|-1\right)_{\#} C\left(\frac{v}{|v|}, t\right) & \text { and } t \in[0,1 / 2], \\
& \text { if } v \in \bar{B}_{2}^{4}(0) \backslash \bar{B}_{1}^{4}(0)
\end{array}\right. \\
&
\end{aligned}
$$

If $v \in \partial \bar{B}_{2}^{4}(0)$, then $\operatorname{supp}(\tilde{C}(v, t)) \subset \partial B_{r(t)}\left(\frac{v}{|v|}\right)$. By the Constancy Theorem there exists $k(v, t) \in \mathbb{Z}$ such that

$$
\tilde{C}(v, t)=k(v, t) \cdot \partial B_{r(t)}\left(\frac{v}{|v|}\right) .
$$

Since $\tilde{C}(v, 1 / 2)=-\operatorname{lk}\left(\gamma_{1}, \gamma_{2}\right) \cdot \partial B_{\pi / 2}\left(\frac{v}{|v|}\right)$, it follows by continuity in the flat topology that $k(v, t)=-\operatorname{lk}\left(\gamma_{1}, \gamma_{2}\right)$ for every $v \in \partial \bar{B}_{2}^{4}(0)$ and $t \in[0,1]$.

By choosing an orientation-preserving homeomorphism $f: I^{4} \rightarrow \bar{B}_{2}^{4}(0)$, we can reparametrize $\tilde{C}: \bar{B}_{2}^{4}(0) \times[0,1] \rightarrow \mathcal{Z}_{2}\left(S^{3}\right)$ to get a map $\Phi: I^{5} \rightarrow$ $\mathcal{Z}_{2}\left(S^{3}\right)$ with the following properties.

4.3. Theorem. Let $\left(\gamma_{1}, \gamma_{2}\right)$ be a 2-component link in $S^{3}$ with $\operatorname{lk}\left(\gamma_{1}, \gamma_{2}\right)=$ -1 . The map

$$
\Phi: I^{5} \rightarrow \mathcal{Z}_{2}\left(S^{3}\right)
$$

obtained by reparametrization of $\tilde{C}$, is continuous in the flat topology and satisfies the following properties:

(1) $\Phi(x, 0)=\Phi(x, 1)=0$ for any $x \in I^{4}$,

(2) $\Phi(x, t)=\partial B_{r(t)}\left(\frac{f(x)}{|f(x)|}\right)$ for every $(x, t) \in \partial I^{4} \times I$,

(3) $\Phi(x, 1 / 2)=\partial B_{\pi / 2}\left(\frac{f(x)}{|f(x)|}\right)$ for any $x \in \partial I^{4}$,

(4) there is no concentration of area:

$$
\lim _{r \rightarrow 0} \sup \left\{\operatorname{area}\left(\Phi(x) \cap B_{r}(p)\right): p \in S^{3}, x \in I^{5}\right\}=0,
$$


MIN-MAX THEORY, WILLMORE CONJECTURE AND THE ENERGY OF LINKS 21

(5) $\sup \left\{\operatorname{area}(\Phi(x)): x \in I^{5}\right\} \leq E\left(\gamma_{1}, \gamma_{2}\right)$,

(6) the center map $Q: \partial I^{4} \rightarrow S^{3}$, given by $Q(x)=\frac{f(x)}{|f(x)|}$, satisfies $\operatorname{deg}(Q)=1$.

Given a link $\left(\gamma_{1}, \gamma_{2}\right)$ in $S^{3}$ with $\operatorname{lk}\left(\gamma_{1}, \gamma_{2}\right)=-1$, it follows from the above properties that the map $\Phi: I^{5} \rightarrow \mathcal{Z}_{2}\left(S^{3}\right)$ satisfies all the assumptions of the $2 \pi^{2}$ Theorem. Therefore there exists $y \in I^{5}$ such that area $(\Phi(y)) \geq 2 \pi^{2}$. Since area $(\Phi(x)) \leq E\left(\gamma_{1}, \gamma_{2}\right)$ for every $x \in I^{5}$, we get $E\left(\gamma_{1}, \gamma_{2}\right) \geq 2 \pi^{2}$. The case $\mathrm{lk}\left(\gamma_{1}, \gamma_{2}\right)=1$ also follows by a change in orientation. In particular this proves the Freedman-He-Wang conjecture.

\section{REFERENCES}

[1] I. Agol, F. C. Marques and A. Neves, Min-max theory and the energy of links arXiv:1205.0825 [math.GT] (2012) 1-19.

[2] F. Almgren, The homotopy groups of the integral cycle groups, Topology (1962), 257299.

[3] F. Almgren, The theory of varifolds. Mimeographed notes, Princeton (1965).

[4] F. Almgren, Some interior regularity theorems for minimal surfaces and an extension of Bernstein's theorem. Ann. of Math. (2) 84 (1966), 277-292.

[5] G. D. Birkhoff, Dynamical systems with two degrees of freedom, Trans. Amer. Math. Soc. 18 (1917), no. 2, 199-300.

[6] W. Blaschke, Vorlesungen Über Differentialgeometrie III, Berlin: Springer (1929).

[7] T. Colding and C. De Lellis, The min-max construction of minimal surfaces, Surveys in Differential Geometry VIII , International Press, (2003), 75-107.

[8] C. B. Croke, Poincaré's problem and the length of the shortest closed geodesic on a convex hypersurface, J. Differential Geom. 17 (1982), no. 4, 595-634 (1983).

[9] M. Freedman, Z-X. He, Z. Wang, Möbius energy of knots and unknots, Ann. of Math. (2) 139 (1994), no. 1, 150.

[10] S. Germain, Recherches sur la théorie des surfaces élastiques, Paris (1921).

[11] Zheng-Xu He, On the minimizers of the Möbius cross energy of links, Experiment. Math. 11 (2002), no. 2, 244-248.

[12] W. Helfrich. Elastic properties of lipid bilayers: Theory and possible experiments. Z. Naturforsch. 28 (1973), 693-703.

[13] D. Kim and R. Kusner, Torus knots extremizing the Möbius energy, Experiment. Math. Volume 2, Issue 1 (1993), 1-9.

[14] B. Lawson, Complete minimal surfaces in $S^{3}$, Ann. of Math. (2) 92 (1970), 335-374.

[15] P. Li and S-T. Yau, A new conformal invariant and its applications to the Willmore conjecture and the first eigenvalue of compact surfaces, Invent. Math. 69 (1982), 269291.

[16] L. A. Lyusternik, A. I. Fet, Variational problems on closed manifolds, (Russian) Doklady Akad. Nauk SSSR (N.S.) 81, (1951). 17-18.

[17] Lyusternik, L.; Schnirelmann, L. Topological methods in variational problems and their application to the differential geometry of surfaces, (Russian) Uspehi Matem. Nauk (N.S.) 2, (1947). no. 1(17), 166-217.

[18] F. C. Marques and A. Neves, Rigidity of min-max minimal spheres in three-manifolds, Duke Math. J. 161 (2012), no. 14, 2725-2752.

[19] F. C. Marques and A. Neves, Min-max theory and the Willmore conjecture, to appear in Ann. of Math. (2013).

[20] H. Poincaré, Sur les lignes godsiques des surfaces convexes, Trans. Amer. Math. Soc. 6 (1905), no. 3, 237-274. 
[21] J. Pitts, Existence and regularity of minimal surfaces on Riemannian manifolds, Mathematical Notes 27, Princeton University Press, Princeton, (1981).

[22] S. D. Poisson, Mémoire sur les surfaces élastiques, Mem. Cl. Sci. Math. Phys., Inst. de France, (1812) 167-225.

[23] J. O'Hara, Energy of a knot, Topology 30 (1991), 241-247.

[24] A. Ros, The Willmore conjecture in the real projective space, Math. Res. Lett. 6 (1999), 487-493.

[25] A. Ros, The isoperimetric and Willmore problems, Global differential geometry: the mathematical legacy of Alfred Gray (Bilbao, 2000), 149-161, Contemp. Math., 288, Amer. Math. Soc., Providence, RI, (2001).

[26] R. Schoen and L. Simon, Regularity of stable minimal hypersurfaces. Comm. Pure Appl. Math. 34 (1981), 741-797.

[27] L. Simon, Lectures on geometric measure theory, Proceedings of the Centre for Mathematical Analysis, Australian National University, Canberra, (1983). vii+272 pp.

[28] F. Smith, On the existence of embedded minimal 2-spheres in the 3-sphere, endowed with an arbitrary Riemannian metric, $\mathrm{PhD}$ thesis supervised by L. Simon, University of Melbourne (1982).

[29] G. Thomsen, Über Konforme Geometrie, I: Grundlagen der Konformen Flächentheorie, Abh. Math. Sem. Hamburg (1923), 31-56.

[30] F. Urbano, Minimal surfaces with low index in the three-dimensional sphere, Proc. Amer. Math. Soc. 108 (1990), 989-992.

[31] T. J. Willmore, Note on embedded surfaces, An. Sti. Univ. "Al. I. Cuza” Iasi Sect. I a Mat. (N.S.) 11B (1965) 493-496.

[32] X. Zhou, Min-max minimal hypersurface in $\left(M^{n+1}, g\right)$ with Ric R $_{g}>0$ and $2 \leq n \leq 6$, arXiv:1210.2112v2 [math.DG] (2012)

Instituto de Matemática Pura e Aplicada (IMPA), Estrada Dona Castorina 110, 22460-320 Rio DE JANEIRO, BRAZIL

E-mail address: coda@impa.br

Imperial College, Huxley Building, 180 Queen's Gate, London SW7 2RH, UNITED KINGDOM

E-mail address: a.neves@imperial.ac.uk 